\title{
DISFUNCIÓN ERÉCTIL DE ORIGEN PSICÓGENO
}

José María Pomerol Monseny.

Instituto de Andrología y Medicina Sexual. Barcelona. España.

Resumen.- OBJETIVO: Revisar los diferentes aspectos relacionados con la disfunción eréctil de tipo psicógeno.

MÉTODO: Revisión de la bibliografía y experiencia personal

CONCLUSIONES: La disfunción eréctil de origen psicógeno es más frecuente de lo que se cree en la actualidad y es posible que aumente en el futuro, sobre todo en hombres jóvenes, en relación con el actual estilo de vida. La causa más frecuente es la ansiedad de rendimiento ligada a diferentes circunstancias. Otras causas vienen dadas por una mala educación sexual, por experiencias sexuales traumáticas durante la infancia, por conflictos en la relación de pareja, por situaciones

CORRESPONDENCIA

José María Pomerol Monseny

Paseo de la Bonanova, 69

08017 Barcelona (España)

jmpomero|@hotmail.com estresantes de cualquier naturaleza o por trastornos psiquiátricos como la depresión entre muchas otras.

La DE psicógena puede asociarse con otros trastornos de la esfera sexual, suele presentarse de forma brusca y es fácil de diagnosticar cuando existen erecciones normales de tipo involuntario o en determinadas circunstancias.

Palabras clave: Disfunción eréctil psicógena. Disfunción eréctil.

Summary.- OBJECTIVES: To review the different aspects of psychogenic erectile dysfunction (PED).

METHODS: To review the bibliography and our personal experience.

CONCLUSION: PED is more frequent than currently thought and may grow in the future, especially in young men, linked to the present style of life. Performance anxiety related to different circumstances is the most common cause of PED. Other causes are given by an inadequate sexual education, traumatic sexual experiences during childhood, a conflictive couple relationship, stressful situations of any nature or psychiatric disorders, like depression, among others.

PED may be associated with other sexual disorders. Usually occurs abruptly and it is easy to diagnose when there are normal erections in any circumstances.

Keywords: Psychogenic erectile dysfunction. Erectile dysfunction.

\section{INTRODUCCIÓN}

La erección es una función de gran complejidad debido a los muchos factores que, a diferentes niveles, intervienen en la misma, siendo algunos de 
ellos todavía desconocidos en la actualidad. En líneas generales, la función eréctil puede verse alterada por patologías que interfieren el control cerebral de la erección, la transmisión de "órdenes neurológicas" a través de la médula espinal o los mecanismos neurovasculares y estructurales peneanos. Una o varias de estas patologías pueden afectar a uno o más niveles del mecanismo eréctil y, a su vez, condicionar el desarrollo de otras que contribuirán, en diferentes grados, a la disfunción eréctil (DE). Todo ello comporta una gran dificultad para conocer con precisión el papel que juegan los distintos factores, ya sean predisponentes, desencadenantes o mantenedores, en la $D E$. Además, las metodologías diagnósticas actuales para valorar los componentes vasculares y neurológicos de la erección son, en muchas ocasiones, poco precisas y fiables por lo que se aplican con menor frecuencia que en el pasado. Ha contribuido también a ello el hecho de no disponer de tratamientos etiológicos adecuados, así como la alta efectividad de los inhibidores de la 5 fosfodiesterasa (iFDE5) como tratamiento sintomático.

Si la determinación de etiologías orgánicas es difícil, todavía lo es más el diagnóstico de factores psicológicos y neurobiológicos relacionados con el control cerebral de la erección.

En el pasado se llegó a creer que la mayoría de las DE eran de origen psicógeno, todo lo contrario de lo que sucede en la actualidad donde se considera que determinadas patologías, condiciones o factores de riesgo cardiovascular (diabetes, hipertensión arterial, hipercolesterolemia, obesidad, fármacos, etc.) constituyen la etiología indiscutible de la $\mathrm{DE}$, sin tener en cuenta la gran cantidad de hombres que, en las mismas circunstancias, no sufren de DE. Es evidente que existen otros factores, ya sean genéticos 0 de cualquier otra naturaleza para explicar el desarrollo del proceso.

Entre los argumentos para defender la existencia de un considerable porcentaje de DE de origen psicógeno, cabe mencionar los resultados obtenidos durante los ensayos clínicos de fármacos para la $D E$ en los que se demuestra que un $20-40 \%$ de los pacientes presentan una buena respuesta, en términos de erección, al placebo utilizado.

\section{ETIOLOGÍA DE LA DISFUNCIÓN ERÉCTIL PSI- CÓGENA}

Una vez realizadas estas consideraciones generales, nos vamos a centrar en los trastornos del control cerebral de la erección. Lo primero que cabe mencionar es la dificultad que existe en la práctica clínica para estudiar los factores constitucionales y biológicos (neurotransmisores, receptores), por lo que, en la actualidad, todavía debemos limitarnos a la valoración y origen de los trastornos psicológicos.

En la DE psicógena pueden existir tanto factores predisponentes (educación restrictiva, abusos sexuales, etc) como precipitantes (experiencias sexuales insatisfactorias, separación de pareja, conflictos económicos, etc.) y mantenedores del proceso (conflictos de pareja, ansiedad de rendimiento, etc.).

Existen pocos estudios científicos que aborden la relación entre la DE y los factores psicógenos. Entre ellos cabe destacar el Massachussets Male Aging Study (1) y el Nacional Health and Social Life Survey (2) en los que se observó una asociación significativa entre la DE y factores psicosociales como síntomas de depresión $(O R=2.88)$, actitudes pesimistas $(O R=3.89)$, perspectivas negativas de la vida $(O R=2.30)$, estrés emocional $(O R=3.56)$ e historia de coacción sexual (OR=3.52).

Existen muchas posibles causas de DE psicógena; las más frecuentes se hallan referidas en la Tabla I. En primer lugar cabe mencionar la ansiedad de ejecución, descrita por Masters y Johnson (3), que presentan determinados hombres después de haber experimentado algún fracaso de la erección. Ante la experiencia vivida, se establece el temor y una absoluta pérdida de confianza respecto a la siguiente relación sexual. El individuo tenderá a la autoobservación del pene, donde focalizará toda su atención, con la consiguiente pérdida del estímulo sexual y la atención hacia la pareja. En estas situaciones, el cerebro libera una mayor cantidad de noradrenalina; su efecto vasoconstrictor sobre las arterias peneanas y el tejido cavernoso puede llegar a ser superior al efecto vasodilatador del óxido nítrico liberado en las terminales nerviosas y vasos peneanos en respuesta al estímulo sexual. La consecuencia es la pérdida de la rigidez peneana, con lo que se establece de manera evidente el importante papel del cerebro en la función sexual. El fracaso de la erección y sus dramáticas consecuencias en la relación sexual con la pareja queda muy "grabado" en el cerebro, pudiendo condicionar los fallos posteriores o la evitación de la actividad sexual.

Con frecuencia, la DE de origen psícógeno coexiste con otros trastornos sexuales como son el deseo sexual inhibido o la eyaculación prematura, pudiendo ser tanto su causa como su consecuencia.

La DE también puede establecerse por factores relacionados con la pareja, ya sea por problemas 
de diálogo o comunicación, por falta de intimidad o de confianza o por conflictos de poder. No siempre es fácil determinar si el origen del problema es la mala relación de pareja o si esta se establece a partir de la disfunción sexual.

Otra presentación frecuente es la pérdida de interés sexual por la pareja, pudiendo evolucionar hacia una verdadera aversión sexual.

Determinadas enfermedades de la pareja, fundamentalmente aquellas que afectan a las zonas erógenas, pueden desencadenar alteraciones del deseo sexual y $\mathrm{DE}$. En procesos crónicos oncológicos o de otra naturaleza, a la afectación psicológica por el problema se suma la inactividad coital durante largos periodos de tiempo. Estas pueden ser las circunstancias del viudo que, al intentar reanudar la actividad sexual con otra pareja, presenta DE. En estos casos, aparte de los aspectos mencionados, puede existir una cierta sensación de culpabilidad y traición a la pareja fallecida, así como temor a la no aceptación de la nueva relación por parte del entorno familiar y social.
En líneas generales, las disfunciones sexuales de la mujer también pueden condicionar disfunciones sexuales en el hombre, de la misma manera que sucede a la inversa, por lo que es fundamental investigar la presencia de las mismas.

Las situaciones de estrés, ligadas a conflictos familiares, laborales, sociales y económicos generan ansiedad. En estas circunstancias se puede perder el interés general por el sexo y la concentración durante el coito, pudiendo desencadenar la $D E$, el abandono progresivo de la actividad sexual y la afectación de la autoestima.

En los últimos años es especialmente preocupante el aumento de la DE entre hombres jóvenes, en los que se pueden detectar problemas relacionados con una educación sexual ausente o restrictiva ligada a actitudes morales y religiosos por parte de los padres o los maestros, así como antecedentes de abusos sexuales en la infancia. La DE también puede ser la consecuencia del actual estilo de vida donde las relaciones sexuales se inician de manera mas precoz que en el pasado y existe una mayor promiscuidad

TABLA I. POSIBLES CAUSAS DE TRASTORNOS PSICÓGENOS EN LA DE.

\begin{tabular}{|l|}
\hline - Ansiedad de rendimiento \\
\hline - Conflictos en la relación de pareja \\
\hline - Problemas en el entorno social y laboral \\
\hline - Problemas afectivos \\
\hline - Estrés \\
\hline - Baja autoestima \\
\hline - Depresión \\
\hline - Inhibición sexual \\
\hline - Consecuencias del abuso sexual durante la infancia \\
\hline - Tabúes religiosos o culturales \\
\hline - Resultado de preferencias sexuales \\
\hline - Miedo al embarazo y enfermedades de transmisión sexual \\
\hline - Neurosis, psicosis \\
\hline - Creencias sexuales erróneas \\
\hline - Consecuencia de otros trastornos sexuales (deseo sexual inhibido, eyaculación prematura o retrasada, etc.) \\
\hline
\end{tabular}


en experiencias tanto hetero como homosexuales. La dificultad para definir las preferencias sexuales puede ser causa de una gran ansiedad y de trastornos tanto del deseo como de la función eréctil. Hombres jóvenes con poca experiencia sexual pueden sentirse cohibidos frente a mujeres de su misma edad que ya han mantenido relaciones con otros hombres, lo que conlleva inseguridad frente a la actividad sexual y afectación de la autoestima.

En la clínica de infertilidad pueden verse parejas que no consiguen la gestación por imposibilidad de realizar la penetración debido a DE psicógena, secundaria al temor inconsciente a la paternidad, ligado generalmente a malas experiencias en el entorno familiar. Estos hombres son capaces de conseguir una erección adecuada por masturbación, pero no lo consiguen en la relación de pareja, fundamentalmente en el momento de realizar la penetración.

Existen una gran cantidad de problemas psiquiátricos que pueden desencadenar una $\mathrm{DE}$. Entre el 50 y el $70 \%$ de los pacientes con depresión presentan trastornos de la esfera sexual $(4,5)$, agravados por el tratamiento antidepresivo $(6,7)$. La depresión es un factor de riesgo para la $D E$, con un riesgo relativo de 1.81 (8), siendo más frecuente en hombres con problemas afectivos (9). Así pues, la relación que existe entre la depresión y la DE es compleja y bidireccional.

En líneas generales, nunca hay que obviar que detrás de cualquier $\mathrm{DE}$, sea cual sea su etiología, existen factores psicológicos que contribuyen a empeorar el problema y muchas veces a mantenerlo a pesar del control y tratamiento de las causas desencadenantes.

\section{ASPECTOS CLÍNICOS}

En la práctica clínica no es difícil detectar una DE psicógena, ya sea generalizada o circunstancial sobre todo cuando el paciente refiere que es capaz de conseguir erecciones normales en el curso de la masturbación o con determinadas parejas, circunstancias o estímulos sexuales. Igual de evidentes son los casos en los que el inicio de la DE coincide con eventos traumáticos como la pérdida de trabajo, problemas económicos, muerte de familiares, etc.

La DE de origen psíquico, al contrario de la de origen orgánico, suele presentarse de forma brusca. La integridad de los mecanismos eréctiles periféricos se establece a partir de la simple observación de erecciones normales involuntarias, ya sea durante la fase REM del sueño, al levantarse por la mañana o frente a determinados estímulos o circunstancias.
En la anamnesis inicial de pacientes con DE es fundamental establecer un clima de confianza para poder detectar todos aquellos factores psicológicos y sociales que hayan podido contribuir en la alteración de la función eréctil. No se precisa ser especialista en trastornos mentales para poder hacerlo; simplemente se precisa de tiempo, saber interrogar de forma adecuada y tener una cierta sensibilidad.

La función del psicoterapeuta debe ser en primer lugar la identificación del problema y las circunstancias desencadenantes. En segundo lugar, ofrecer todo el apoyo psicológico para que el paciente pueda disminuir la ansiedad y convivir con la DE y todas sus consecuencias. Finalmente, el psicoterapeuta debe aplicar todas las metodologías precisas para resolver la $\mathrm{DE}$, con ayuda o no de tratamiento médico sintomático.

\section{BIBLIOGRAFÍA y LECTURAS RECOMENDADAS (*lectura de interés $y^{* *}$ lectura fundamental)}

*1. Feldman HA, Goldstein I, Hatzichristou DG, et al. Impotence and its medical and psychosocial correlates: Results of the Massachusetts Male Aging Study. J Urol 1994; 151 L:54-61.

*2. Lazarus AA. The treatment of a sexually inadequate man. En Ullmann LP, Drasner L (eds): Case Studies in Behavior Modification. New York, Holt, Rinehart, and Winston, 1965, p 243-260.

*3. Masters WH, Johnson VE. Human Sexual Inadequacy. Boston, Little Brown, 1970.

*4. Angst J. Sexual problems in healthy and depressed persons. Int Clin Pharmacol 1998;13(Suppl 6):S1-S4.

*5. Bonierbale M, Lançon C, Tignol J. The ELIXIR study: evaluation of sexual dysfunction in 4557 depressed patients in France. Curr Med Res 2003; $19: 114-24$.

*6. Balon R, Yeragani VK, Pohl R, Ramesh C. Sexual dysfunction during antidepressant treatment. J Clin Psychiatry 1993;54:209-12

**7. Montejo-Gonzalez AL, Llorca G, Izquierdo JA, et al. SSRI-induced sexual dysfunction: fluoxetine, paroxetine, sertraline and fluvoxamine in a prospective multicenter and descriptive clinical study of 344 patients. J Sex Marital Ther 1997;23:176-94

**8. Araujo AB, Durante R, Feldman HA, et al. The relationship between depressive symptoms and male erectile dysfunction: prospective results from the Massachusetts Male Ageing Study. Am J Epidemiol 2000;152:533-41

*9. Mitchell JF, Popkin M. Antidepressant drug therapy and sexual dysfunction in men: a review. J Clin Psychopharmacol 1983;3:276-9 